\title{
Arquitetura Dirigida a Modelos (MDA): Utilizando Modelos no Desenvolvimento de Sistemas
}

\section{Resumo}

A rede de computadores, uma tecnologia com trinta anos de idade, está promovendo um grande impacto nas pessoas e instituições incluindo o governo, negócios e educação. A internet, a intranet e a tecnologia emergente das redes de dispositivos móveis são exemplos de redes de computadores.

Elas permitem a implementação de sistemas distribuídos onde componentes localizados na rede podem comunicar e coordenar suas ações através da troca de mensagens. $\bigcirc$ objetivo básico dos sistemas distribuídos é o compartilhamento de periféricos, recursos (mecanismos de busca) e de informações (dados de um banco de dados distribuídos, vídeos, músicas etc.). Neste ambiente de processamento, os sistemas isolados e monolíticos estão sendo substituídos por sistemas compostos por elementos ativos ou passivos inseridos em uma nova rede de serviços.

A evolução deste ambiente de processamento provocou o surgimento de novas tecnologias de desenvolvimento de sistemas e de linguagens de programação. A evoluçãoé tão rápidaque sistemas em produção desenvolvidos há um ou dois anos tornam-se obsoletos devendo ser adaptados às novas tecnologias e até substituídos. $\mathrm{O}$ mesmo ocorre com as equipes de desenvolvimento que são obrigadas a atualizarem seus conhecimentos com a mesma velocidade.

A MDA (Arquitetura Dirigida a Modelos) é uma tecnologia desenvolvida pelo Object Management Group (OMG) que consiste em promover o desenvolvimento de sistemas a níveis mais altos de abstração de tal forma que os modelos desenvolvidos fiquem independentes da plataforma de implementação. A seguir estes modelos são transformados em modelos dependentes da plataforma de implementação usando-se padrões de projeto e frameworks. $\mathrm{O}$ trabalho de transformação de modelos é feito usando-se ferramentas de transformação de modelos e de geração de parte do código da aplicação.

Nestas condições não será necessário adaptar os sistemas em produção a novas tecnologias: basta repetir a geração de modelos agora dependentes da nova plataforma.

\begin{abstract}
The computer network, a thirty year old technology, is promoting a major impact on individuals and institutions including government, business and education. The internet, intranet and the emerging technology of mobile devices network are examples of computer networks.

They allow the implementation of distributed systems where components located at networked computers communicate and coordinate their actions only by routing packets. The basic goal of distributed systems is the sharing of peripherals, resources (search engines) and information (data of shared database, videos, music, etc.). In this environment, isolated and monolithic systems are being replaced by systems composed of active or passive elements inserted in a new services network.

The evolution of this processing environment has induced the emergence of new technologies, development of systems and programming languages. The evolution is so rapid that production systems developed in less than two years are becoming obsolete and hence must be adapted to new technologies or even replaced. The same happens with the
\end{abstract}

\footnotetext{
${ }^{1}$ Mestre em Engenharia. Professor e Coordenador do curso de Sistemas de Informação das Faculdades Integradas "Campos Salles".
} 
development teams that are required to update their knowledge with the same rhythm.

The MDA (Model - Driven Architecture) is a technology developed by the Object Management Group (OMG), meant to promote the development of systems to higher levels of abstraction so that the models are developed independent of the platform of implementation. Below these models are transformed into models dependent on the platform of implementation using design patterns and frameworks. The work of transformation of the models is done using tools of transformation of models and generation of the code of the application.

In this way it will not be necessary to adapt the systems in production to new technologies: just repeat the generation of models now dependent on the new platform.

\section{1- Introdução}

Uma metodologia de desenvolvimento de software é em geral baseada no ciclo de vida do desenvolvimento de um sistema e está composta por um conjunto de estágios de desenvolvimento. A metodologia descreve quais são os trabalhos a serem desenvolvidos em cada estágio, documentos necessários para a entrada em cada estágio e quais são os documentos produzidos por cada estágio. Basicamente a metodologia define um plano para a produção de um sistema.

A construção de sistemas grandes e complexos é um trabalho longo e complicado, envolvendo o desenvolvimento de vários serviços, a maioria interdependentes. Usando uma metodologia, é possível gerenciar o desenvolvimento do sistema permitindo o planejamento, controle e monitoração do processo de desenvolvimento.

Ao longo dos anos surgiram várias metodologias cada uma definindo uma visão de como se constrói um determinado tipo de sistema. No caso dos sistemas de informação têm-se metodologias que dão ênfase ao fluxo de dados no interior do sistema, outras na estruturação e relacionamento dos dados armazenados etc.

As metodologias estão em constante evolução a fim de absorver os avanços tecnológicos e as novas idéias para o desenvolvimento de siste- mas. Por exemplo, no início dos anos 60 foram feitos os primeiros trabalhos de simulação em computadores: neste tipo de aplicação se tem vários "agentes" independentes capazes de responder a eventos externos individualmente. Era possível modelar cada agente individualmente, porém era muito difícil modelar um conjunto de agentes interagindo entre si ao mesmo tempo.

Este tipo de aplicação é muito difícil de ser programado usando-se linguagens procedurais de terceira geração, porque elas estão baseadas na seguinte hipótese: a estrutura do programa controla o fluxo de execução.

Uma solução para este problema foi a criação das primeiras linguagens de programação (linguagem Simula - projeto iniciado em 1962) que permitiram estruturar os programas como sendo um conjunto de agentes de software independentes como ocorre nas aplicações de simulação da realidade.

Por outro lado, nos anos 80 e 90 ocorreu um grande desenvolvimento das interfaces gráficas (GUI - Graphical User Interface): estas interfaces trouxeram problemas parecidos com os problemas de simulação para o desenvolvimento de software porque as interfaces gráficas permitiam muitas alternativas de ações ao mesmo tempo. Cada ação por sua vez definia novas opções de tal forma que era quase impossível para o projetista do sistema saber qual seqüência de opções seria adotada pelo operador ao usar uma interface gráfica.

Nestas condições, a grande maioria das aplicações desenvolvidas com o uso de interfaces gráficas são muito difíceis de serem implementadas usando-se linguagens procedurais, o que provocou a reavaliação dos paradigmas usados na definição das linguagens de programação e o surgimento das linguagens orientadas a objetos como $\mathrm{C}++$, Java, Visual Basic.NET, Ada, Perl etc.

As linguagens de programação evoluíram no sentido de aumentar seu poder de processamento e de abstração: em vez de usar instruções ao nível de hardware, como era feito antigamente, agora os programadores podem especificar dados e algoritmos em função do domínio do problema. 
Sob o ponto de vista do hardware verificase que a Lei de Moore é obedecida com relativa precisão. Essa lei foi proposta em 1965 pelo engenheiro Gordon Moore, co-fundador da Intel, e estabelece que "a densidade de transistores nos circuitos integrados duplica a cada dois anos". A conseqüência desta evolução rápida é que os aparelhos que os contêm tornam-se cada vez mais pequenos, rápidos e potentes.

Este rápido crescimento da capacidade computacional das máquinas e seu baixo custo resultaram na demanda por sistemas cada vez mais complexos e distribuídos, o que resultou na reavaliação da forma de se desenvolver sistemas principalmente a partir dos anos 90 . O surgimento da internet também é uma conseqüência desta nova realidade.

Atualmente têm-se várias tecnologias para a implementação de sistemas distribuídos de qualquer porte. Estas tecnologias podem ser agrupadas em duas grandes plataformas:

\section{J2EE (Java 2 Enterprise Edition) Microsoft.NET}

Estas tecnologias permitem substituir os sistemas isolados e monolíticos por sistemas compostos por elementos ativos ou passivos inseridos em uma rede de serviços. Em geral se usa o conceito de componentes que podem ser combinados a fim de atender a novos requisitos do sistema de informação da empresa.

A plataforma J2EE define os seguintes componentes a serem usados no serviço de implementação de um sistema distribuído:

- Cliente de aplicativos e applets (programas que são executados pelos navegadores - browsers). São componentes a serem executados na máquina cliente (computador - micro de mesa, notebook, smartphone, celular, micro de mão (ipaq) etc), conectado a um computador servidor).

- Componente da tecnologia Sevlet e JSP (Java Server Page). São componentes a serem executados no servidor.

- Componentes EJB (Enterprise Java Beams).
São componentes de negócio que são executados no servidor.

As componentes J2EE são escritas na linguagem Java.

Da mesma forma a plataforma.NET define um conjunto de componentes a serem usados na implementação de sistemas distribuídos.

- Componentes WFP (Windows Presentation Foundation): são componentes a serem executados na máquina cliente.

- WCF (Windows Communication Foundation): são componentes usados para a comunicação entre aplicações no ambiente distribuído.

- Componentes (WF): permitem a criação de workflows em qualquer aplicação.

- Componentes ASPNET: permitem o desenvolvimento de sistemas web.

Diferentemente da plataforma J2EE que permite somente o uso da linguagem Java na implementação dos programas que compõem um sistema, na plataforma.NET pode-se optar por uma das linguagens: C\#, Visual Basic.NET, $\mathrm{VC}++$ gerenciado, J\#, Delphi 8.NET, C\# Builder, Cobol.NET e Visual Perl.NET. Para as aplicações web tem-se o ASSP.NET e os web services (programa que presta "serviços" para outras máquinas conectadas à internet).

A evolução da tecnologia nestas duas plataformas é muito grande tornando obsoletos sistemas que estão sendo utilizados (em produção) há dois anos ou menos o que provoca a sua adaptação à nova versão da tecnologia ou sua substituição por outro sistema mais moderno.

\section{2- O Desenvolvimento de Software}

Esta evolução rápida da tecnologia provoca os seguintes problemas no desenvolvimento de sistemas:

- Produtividade

- Portabilidade

- Manutenção e documentação

- Interoperabilidade 


\subsection{Produtividade}

O processo de desenvolvimento de software atual é quase artesanal, isto é, voltado para o projeto/código de baixo nível. Basicamente têmse as seguintes fases: definição de requisitos, análise, projeto, codificação e implementação. A documentação é produzida nas três primeiras fases com o uso do ciclo de vida incremental e interativo ou com o ciclo de vida tradicional (em cascata). No modelo orientado a objetos, por exemplo, ela é composta pelas descrições dos casos de uso, diagramas de classes, diagramas de seqüência para todos os casos de uso etc. Em geral o volume de papel é muito grande sem que se tenha escrito uma linha de código.

A programação extrema (XP - eXtreme programming) propõe a redução extrema da documentação, porém ela resolve somente parte do problema: certamente a equipe que desenvolve o sistema conhece os seus requisitos e o que deve ser feito para atendê-los. Quando o sistema entra em produção, provavelmente a manutenção será feita por outra equipe: o fato da documentação ser mínima implicará em um tempo muito grande para se entender como o sistema foi implementado e a partir daí executar a manutenção. A situação é equivalente a de uma pessoa que tem que se deslocar em uma cidade, que ela não conhece, sem ter o seu mapa.

O trabalho nestas condições tem uma baixa produtividade, porque o nível de abstração é muito baixo.

\section{2- Portabilidade}

A cada ano surgem novas tecnologias que são adotadas pelas empresas pelos seguintes motivos:

- exigência dos clientes (web services é um exemplo típico);

- elas, em geral, resolvem as deficiências das tecnologias que estão em uso;

- as empresas que fornecem as ferramentas de desenvolvimento que estão em uso cancelam o suporte em função das novas tecnologias, por exemplo, a UML substituiu o OMT.

Tudo isso implica em sérios problemas de portabilidade dos sistemas que estão em produção. Em geral eles são adaptados à nova tecnologia ou reescritos: as duas soluções implicam na perda do investimento feito para desenvolvê-los e em novos custos para a sua implementação usando novas tecnologias.

\section{3- Manutenção e documentação}

A documentação de um sistema sempre foi um ponto fraco da metodologia usada em seu desenvolvimento. Existem dois fatores importantes quando se consideram os problemas de documentação de um sistema:

- em geral existe pouco interesse no seu desenvolvimento;

- a cada manutenção feita no sistema deveria corresponder uma mudança na documentação o que dificilmente é feito.

Uma solução seria o uso de uma ferramenta que promovesse a documentação automática, a partir do código fonte (como existe na plataforma Java), o que resolveria o problema de documentação em baixo nível. No caso de sistemas complexos a documentação a níveis mais altos é essencial, permanecendo, neste caso, os problemas de documentação.

\section{4- Interoperabilidade}

Os problemas de interoperabilidade vêm do seguinte fato: nenhum sistema opera isoladamente. Um exemplo típico são as aplicações web: elas rodam usando um navegador (browser) e são desenvolvidas usando-se várias tecnologias tais como XHTML, ASP, JSP etc. e acessam informações contidas em um sistema gerenciador de banco de dados, isto é, deve haver interoperabilidade entre estes dois sistemas. 


\section{3- O que é a MDA}

A MDA é uma tecnologia desenvolvida pela OMG (Object Management Group) que permite minimizar os problemas apontados anteriormente. Basicamente ela propõe o desenvolvimento de sistemas a níveis mais altos de abstração de tal forma que os modelos produzidos fiquem independentes da plataforma de implementação. A seguir estes modelos são transformados em modelos dependentes da plataforma de implementação usando-se padrões de projeto e frameworks. $O$ trabalho de transformação de modelos é feito usando-se ferramentas de transformação de modelos e de geração de parte do código da aplicação.

A história do desenvolvimento de software é a história da elevação do nível de abstração. À medida que o nível de abstração aumenta são desenvolvidas ferramentas para fazer o mapeamento de um nível para o seguinte automaticamente. Atualmente as aplicações são escritas em uma linguagem de alto nível que pode ser mapeada automaticamente para uma linguagem de nível inferior.

Em geral este nível inferior não corresponde à linguagem assembler. A Figura 3.1 mostra a evolução do nível de abstração.

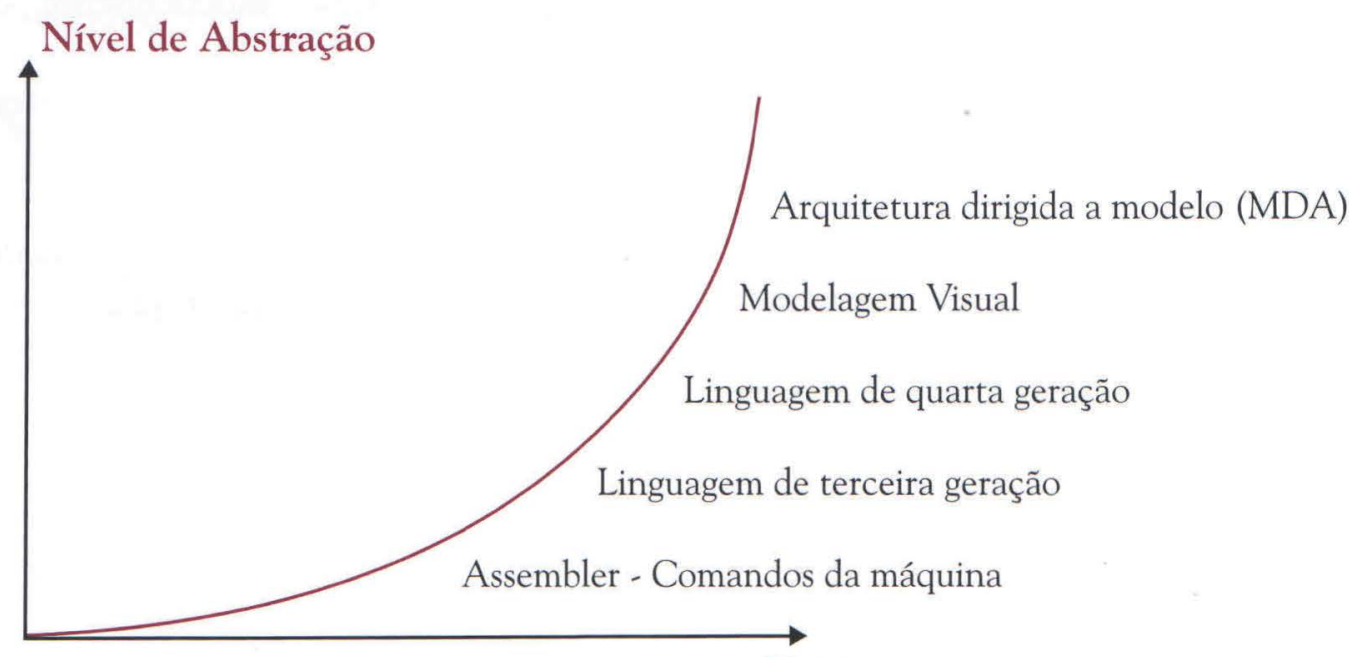

Figura 3.1 - Elevação do nível de abstração Tempo

Fonte: documentação da ferramenta case Ameos

A partir de modelos independentes de plataformas de software ou de projetos de arquitetura de software é possível transformar estes modelos para cada nova plataforma que for proposta com o desenvolvimento da tecnologia sem que se perca o investimento feito no desenvolvimento dos sistemas que estão em produção.

\subsection{O que é um modelo}

No dicionário têm-se várias definições para a palavra modelo:

- Um exemplo usado por um artista.
- Uma pessoa posando para um artista.

- Réplica de um item construído com escala menor (maquete).

Todas as definições acima têm em comum os seguintes aspectos:

- o modelo ser sempre uma abstração de alguma coisa que existe na realidade;

- o modelo é diferente daquilo que ele representa, isto é, os detalhes são omitidos ou o tamanho é diferente. $\mathrm{O}$ modelo pode ser usado como um exemplo para produzir alguma coisa que existe na realidade. 
Um modelo é sempre escrito em uma linguagem podendo ser usadas as linguagens UML, português, linguagem de programação ou qualquer outro sistema. Um modelo descreve um sistema com mais detalhes do que outro ou com uma visão diferente. Observar que os modelos propostos pela MDA são direcionados para um tipo de relacionamento entre modelos: gerar um modelo automaticamente a partir de outro modelo.

\section{2- Tipos de modelos}

Existem vários tipos de modelos e várias formas de diferenciar os mesmos. Cada modelo é baseado na resposta feita a uma pergunta. Cada resposta varia de acordo com as circunstâncias.

Exemplos:

- Em qual fase do desenvolvimento do software o modelo será usado? É um modelo de análise ou projeto?

- O modelo contém muitos detalhes? Ele é abstrato ou detalhado?

- Qual é o sistema que o modelo descreve? Um modelo de negócio ou modelo de software?

- Quais são os aspectos que o modelo descreve? É um modelo estrutural estático ou dinâmico?
- O modelo é específico para determinada tecnologia? Ele é dependente ou independente da plataforma?

- Qual é a plataforma que suporta o modelo? É o EJB, MER, C++, XML ou outro modelo?

As diferenças entre os modelos são importantes no contexto das transformações de modelos. Para considerar um modelo de análise ou projeto é necessário saber o que é análise e projeto no desenvolvimento de um sistema. Da mesma forma para identificar um modelo abstrato ou detalhado depende daquilo que se considera detalhe.

Basicamente a MDA propõe os seguintes modelos:

- Modelo de negócio ou independente do ambiente de computação (CIM).

- Modelo Independente da plataforma (PIM).

- Modelo específico para determinada plataforma (PSM).

Na Figura 3.2 têm-se os modelos propostos pela MDA e sua hierarquia.
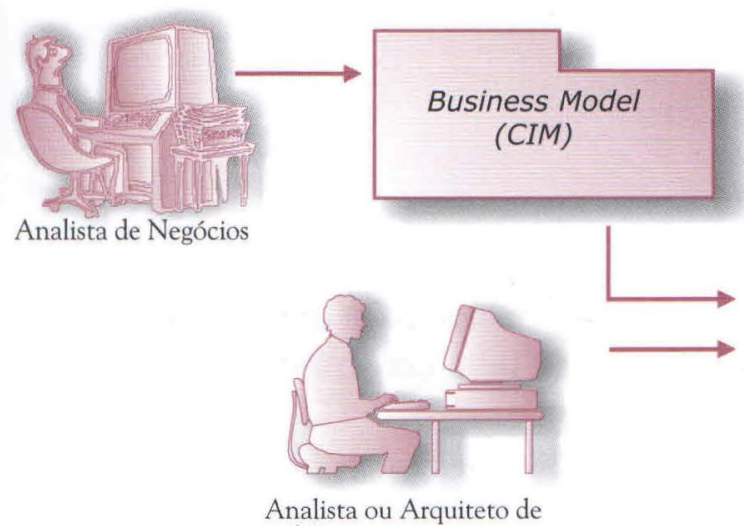

Platform Independent

Analysis Model (PIM)

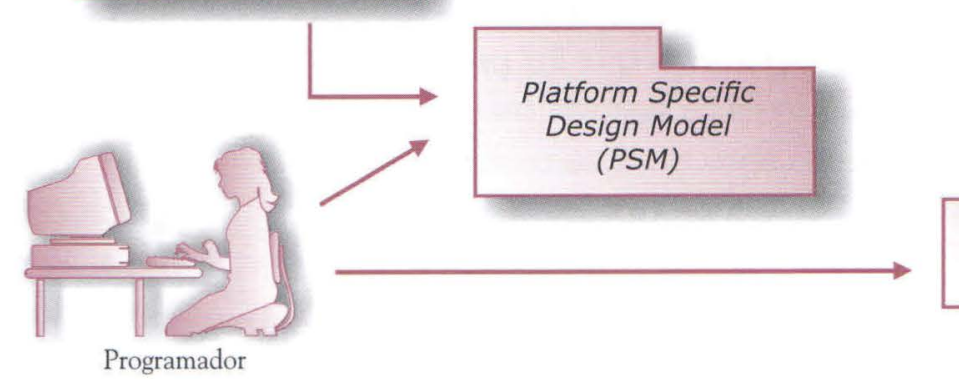

Figura 3.2. Modelos propostos pela MDA e sua hierarquia Fonte: Which MDA Tools are Right for you? : Rosen 
O modelo independente do ambiente de computação (CIM), ou modelo do negócio, representa o sistema usado pela empresa no seu funcionamento. Um modelo de software é desenvolvido quando se decide que parte do modelo de negócio será suportado por um software. Portanto, é impossível a montagem automática de um PIM a partir de um CIM, porque a decisão sobre a informatização é sempre feita por uma pessoa e não por uma ferramenta de transformação de modelos.

O padrão MDA define os conceitos de PIM e PSM, mas é difícil definir os limites entre eles na prática. Eventualmente, pode-se afirmar que um modelo é mais dependente da plataforma e outro menos dependente.

\section{4- Transformação}

Um software ou ferramenta de transformação é uma aplicação projetada para converter automaticamente um PIM para um PSM de acordo com regras de transformação definidas com precisão. Outra ferramenta gera parte do código a partir do PSM.

Uma transformação está composta por um conjunto de regras de transformação, isto é, pode-se ter um conjunto de regras de transformação de UML para C\# e outro de UML para VB.NET. Nestas condições uma transformação corresponde a um conjunto de regras de transformação que descrevem como um modelo expresso na linguagem fonte pode ser convertido em um modelo expresso na linguagem selecionada para o projeto do sistema.

$\mathrm{Na}$ figura 4.1 tem-se um PIM composto pela classe Cliente.

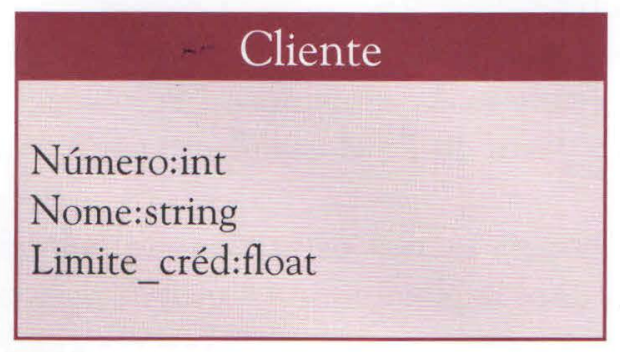

Figura 4.1. PIM composto pela classe Cliente
No PSM para a plataforma J2EE, todos os atributos são transformados para variáveis de instância encapsuladas de acordo com a transformação:

a) para cada classe chamada $<$ nome da classe $>$ no PIM deve-se ter uma classe chamada $<$ nome da classe $>$ no PSM;

b) para cada atributo público chamado $<$ nome do atributo: tipo > do PIM deve-se ter no PSM os seguintes atributos e operações:

1) uma variável de instância privada com o mesmo nome do atributo;

2) uma operação pública cujo nome é composto pelo prefixo get concatenado com o nome do atributo e o tipo do dado retornado;

3) uma operação pública cujo nome é composto pelo prefixo set concatenado com o nome do atributo. $\mathrm{O}$ argumento deve ser o nome do atributo com o respectivo tipo. Não retorna valores.

Na Figura 4.2 tem-se o PSM para J2EE segundo esta transformação.

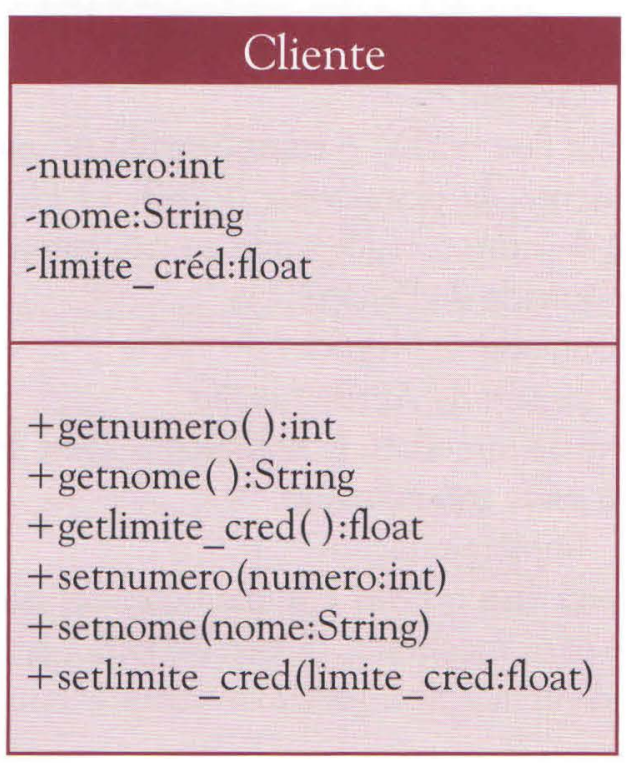

Figura 4.2 . PSM para a plataforma J2EE 


\section{Incorporando a MDA em uma} Metodologia de Desenvolvimento de Sistemas

Toda metodologia de desenvolvimento de sistema está composta pelas seguintes fases:

a) Identificação de Requisitos:

Nesta fase pretende-se construir o modelo de negócios do sistema [CIM] onde são descritos os requisitos do sistema em termos de objetivos e metas, recursos, termos e regras do negócio e processos.

Em geral usam-se textos acompanhados de descrições simplificadas dos casos de uso e o diagrama de atividades. Basicamente neste trabalho são definidas as seguintes visões:

- Dados - esboço do Diagrama de Classes

- Processos - esboço dos Diagramas de Atividade

- Requisitos Funcionais - esboço do Diagrama de Caso de Uso

b) Análise:

Nesta fase o modelo de negócios é transformado em modelo do sistema de informação, isto é, os processos são transformados em casos de uso e os termos em classes. Os requisitos são definidos com precisão e são construídos os modelos de domínios que irão compor o PIM.

c) Projeto:

Nesta fase o PIM é transformado no modelo dependente da plataforma (PSM). O PSM em geral é descrito considerando-se três camadas:

- Camada de Persistência

- Camada de Negócio

- Camada de Apresentação

Deve-se escolher a tecnologia para implementar estas três camadas.

d) Implementação:

Nesta fase o PSM é implementado, isto é, o código para as camadas de persistência e apresentação da aplicação é gerado automaticamente.

\subsection{Uso de ferramentas}

A atividade mais importante na MDA é a transformação entre modelos. Em algumas fases do desenvolvimento do sistema pode-se optar pelo uso das ferramentas de transformação.

Um recurso importante da ferramenta de transformação é a possibilidade de alterar as regras de transformação para que elas possam ser moldadas para as necessidades específicas de determinado projeto. Uma ferramenta com estas características tende a reduzir a maior deficiência dos geradores de código: produzir um programa muito extenso e ineficiente.

As principais ferramentas disponíveis no mercado atualmente são:

- Optimalj;

- Ameos (teve seu código aberto a partir de julho/2007);

- AndroMDA (ferramenta gratuita);

- Borland Together Architect 2006.

\subsection{Geração automática do código}

Como no caso dos modelos, boa parte do código é gerado a partir de padrões e frameworks.

Um padrão de programação (ou de projeto) resolve determinada necessidade de codificação. Considere a aplicação "Sistema para o Controle de Pedido". Toda empresa usa um sistema deste tipo moldado para as suas necessidades, porém este tipo de sistema tem características comuns.

Um Framework é um sistema que implementa estas características comuns. Nestas condições pode-se desenvolver um sistema a partir de um Framework. Assim, um padrão de codificação/ Projeto é mais genérico e abstrato do que um Framework.

Basicamente um Framework pode ser desenvolvido usando-se um conjunto de padrões de codificação/Projeto.

A codificação da aplicação é feita a partir dos seguintes elementos:

- uso de Frameworks genéricos e estáticos;

- Frameworks dinâmicos (da aplicação) gerados a partir do PSM usando-se padrões de código;

- código escrito manualmente pelo programador para implementar a lógica da aplicação.

Um dos Frameworks genéricos mais utilizados é o Apache Struts para a camada de apresentação. Em geral eles são instalados junto com as 
ferramentas na forma de arquivos com a extensão. jar.

Os Frameworks dinâmicos dependem da aplicação, isto é, do PSM gerado. Se as classes do PSM forem alteradas eles também sofrerão modificações.

O Framework dinâmico usa padrões de programação e o Framework estático. Em geral seu código não pode ser editado.

\section{6- Desenvolvimento de um Sistema Usando a Ferramenta OPTIMALJ}

Neste trabalho pretende-se apresentar o desenvolvimento do software "Sistema Gerencial de Representação Comercial" usando-se a ferramenta OptimalJ que implementa os conceitos da MDA. Este projeto foi desenvolvido pela aluna Vanessa Bessa Rodrigues no TCC "Princípios de Arquitetura Orientada a Modelos" sob minha orientação.
A ferramenta Optimalj tem os seguintes recursos:

- suporte a Metamodelos de Domínio, Aplicação e Código. Uso de padrões de Projeto/Código e Frameworks (Apache Struts). Modelos de persistência EJB e DAO, Web Services;

- suporte para todos os Diagramas da UML.

O mesmo software foi desenvolvido, usando-se o Processo Unificado (PU), pelas alunas Priscilla Hermanas da Silva e Simone Santos da Cruz no TCC "Sistema Gerencial de Representação Comercial" nas Faculdades Integradas "Campos Salles".

6.1- Desenvolvimento do modelo do negócio

A documentação deste sistema descreve a montagem do modelo de negócio: este modelo foi transformado resultando o PIM do sistema que corresponde ao diagrama de classe.

Na Figura 6.1 tem-se o PIM desse sistema. 


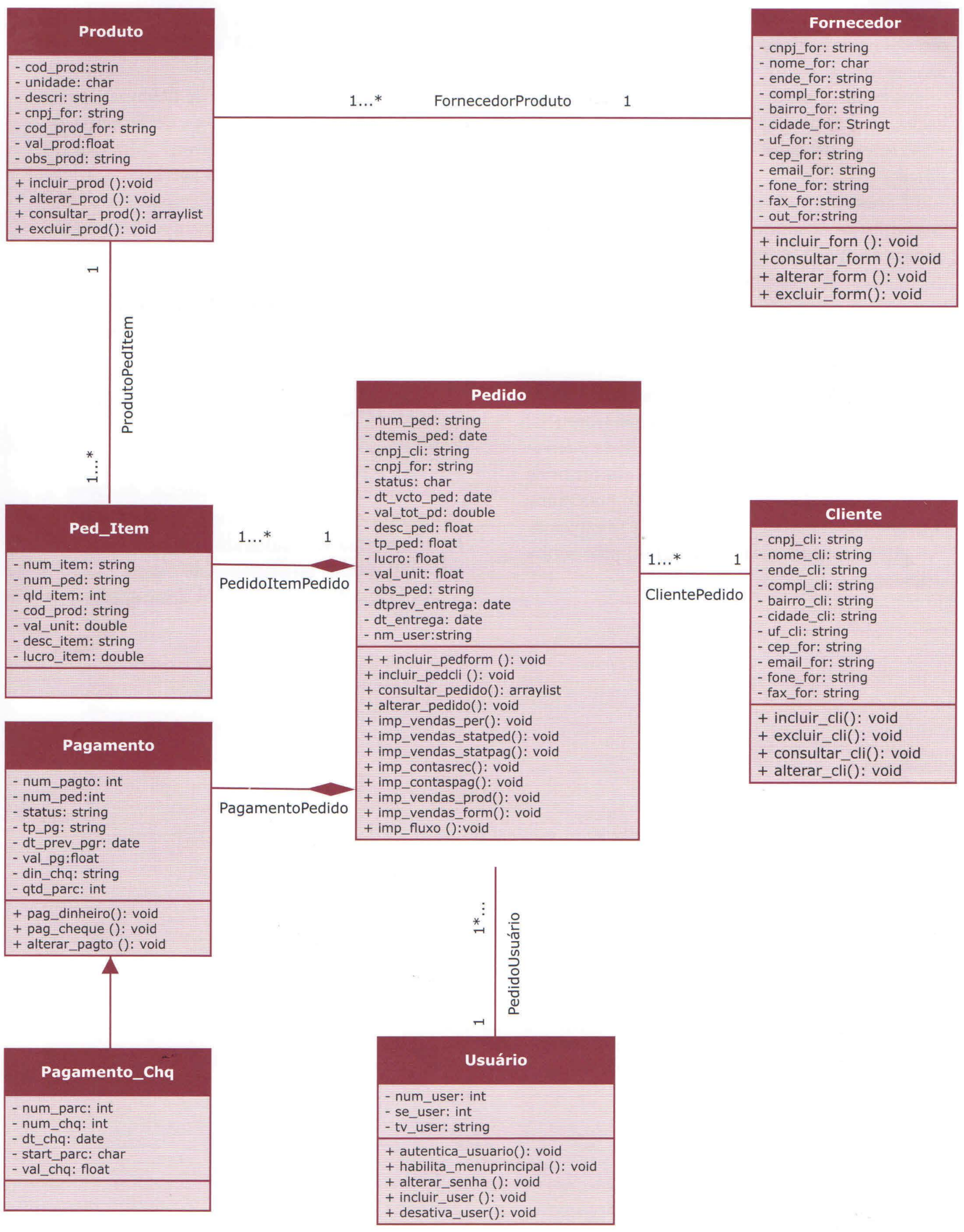

Figura 6.1- PIM do Sistema Gerencial de Representação Comercial 


\section{2- Geração do PSM}

Considerando que cada camada será implementada usando tecnologias diferentes, serão necessários três modelos PSM:

a) modelo entidade/ relacionamento porque a persistência será implementada usando-se um sistema gerenciador de banco de dados relacional;

b) a segunda camada será implementada usando-se um modelo EJB (Enterprise Java Beans).
Ele será expresso em uma linguagem gráfica que é uma extensão da UML: nele são representadas as classes, suas associações etc., porém têm-se vários estereótipos definidos para representar detalhes específicos da plataforma EJB;

c) a primeira camada será implementada usando-se a linguagem HTML e a tecnologia JSP (Java Serve Page).

Na Figura 6.2 têm-se os modelos a serem gerados a partir do PIM.

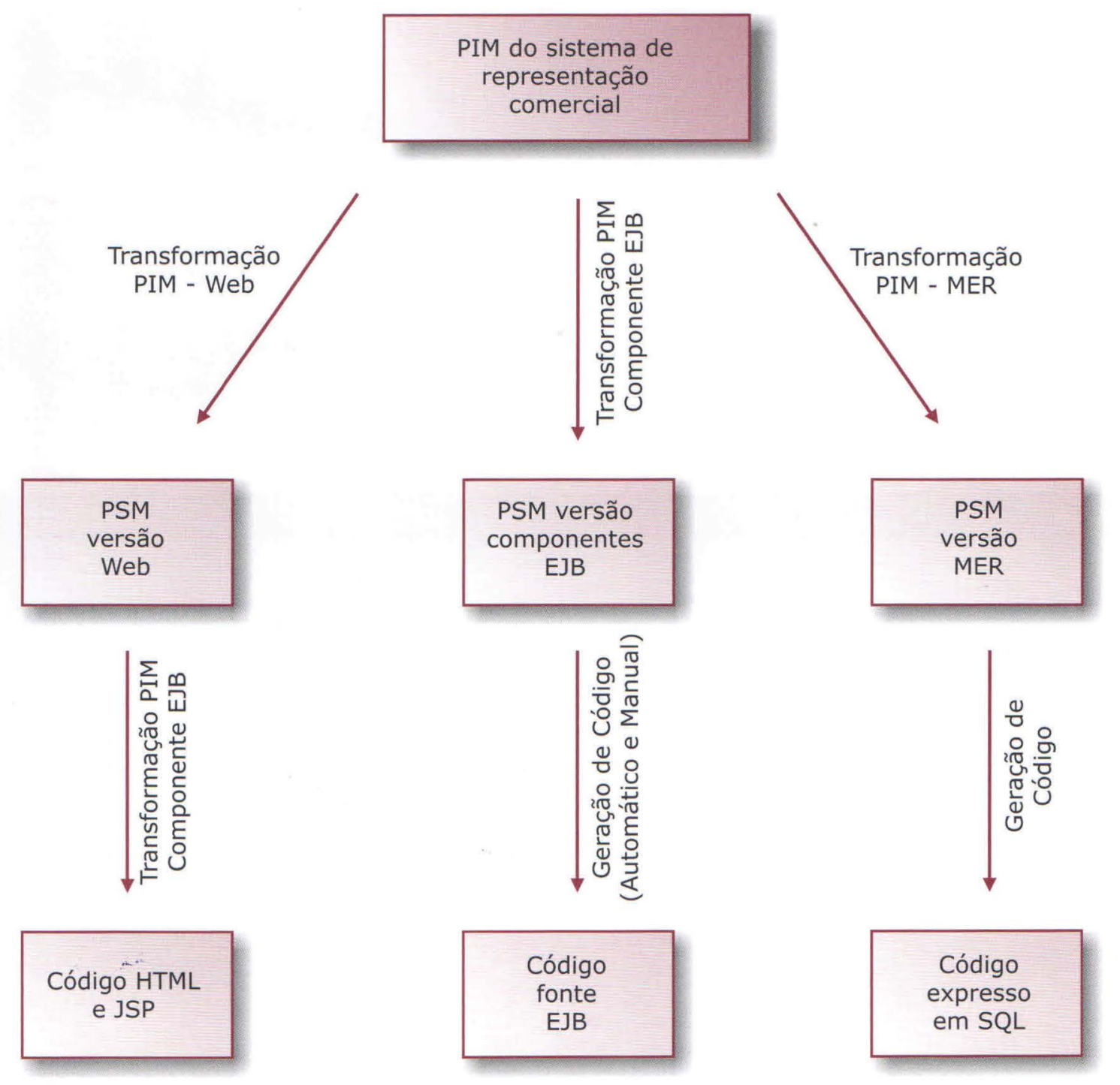

Figura 6.2. Modelos do Sistema de Representação Comercial gerados a partir do PIM 
6.3- Execução da aplicação: testes

Para executar a aplicação deve-se antes ativar o servidor da aplicação e o servidor web.
A seguir é iniciada a execução da aplicação: ela imprime a página de apresentação do sistema (Figura 6.3).

\section{PedidoWeb}

Components

Show ClienteSvc

Show FornecedorSvc

Show PagamentoSvc

Show Pagamento chqSvc

Show Ped ItemSvc

Show PedidoSvc

Show ProdutoSvc

Show UsuarioSve
Welcome to our Web site.

\section{pedidoWeb}

Figura 6.3- Página principal do sistema.

Fonte: Ferramenta OptimalJ 
Figura 6.4- Página gerada acesso a tabela PEDIDO

Fonte: Ferramenta OptimalJ

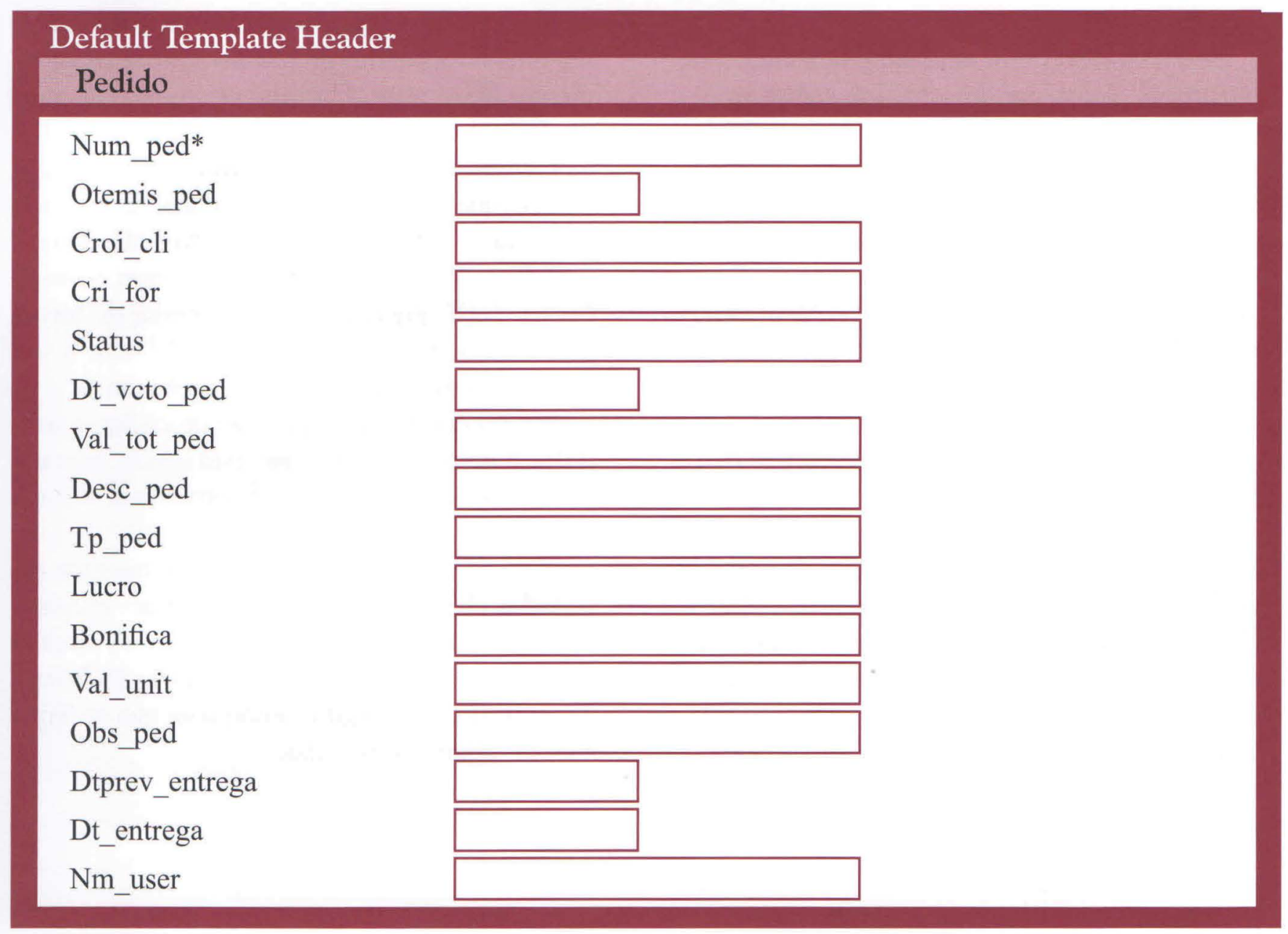

Na Figura 6.4 tem-se a página para cadastrar um pedido.

\subsection{Modificando as páginas geradas} automaticamente

As páginas geradas automaticamente podem ser usadas para efeito de testes, entretanto a aplicação será distribuída usando uma interface projetada para atender aos requisitos do sistema.

Nestas condições, a ferramenta permite que o endereço apresentado na Figura 6.4 seja associado a outra página que contém o projeto da interface feito pélo desenvolvedor.

\section{5- Distribuição da Aplicação}

Feitos todos os testes a aplicação pode ser distribuída (colocada em produção). Pode-se criar um pacote de instalação da aplicação selecionando no menu principal:

\section{GENERATE ALL $>$ ARCHIVES}

A principal vantagem que se obtém com o uso de pacotes de instalação é que a aplicação fica registrada no Windows podendo ser ativada a partir do menu iniciar.

Caso seja necessário desinstalar, pode executar este serviço a partir do Windows clicando-se nas opções:

PAINEL DE CONTROLE $>$ ADICIONAR OU REMOVER PROGRAMAS 


\section{7- Conclusões}

A MDA representa uma mudança radical de paradigma, isto é, dar inicialmente ênfase ao negócio (produzir o CIM) e depois criar os modelos independentes da plataforma (PIM) para cada sistema da empresa.

A partir do PIM usar ferramentas para a montagem dos modelos dependentes da plataforma (PSM) e geração parcial do código. Além destas mudanças algumas ferramentas case como o OptimalJ e o Ameos permitem criar um PIM a partir de PSM's o que é muito útil para a atualização ou substituição de sistemas ligados a um custo reduzido.

Muitos profissionais têm dúvidas a respeito da geração automática de modelos e de parte do código, afirmando que os resultados não são eficientes. Entretanto, eles não avaliam o resultado da tradução de um código fonte para a linguagem de máquina ou bytescodes gerados pela "compilação" de um código escrito em Java. Basicamente o programa executável é gerado a partir do código fonte.
No ambiente Microsoft está se observando um amadurecimento no processo de desenvolvimento de software. O ganho em termos de tempo de desenvolvimento é substancial.

O Framework.NET 3.5 possui a maioria das rotinas que os programadores eram obrigados a codificar com freqüência, evitando o clássico retrabalho. Além disso, ferramentas com o visual Studio. NET preocupam-se em gerar, de forma automática, todo o código que não tem a ver com o desenvolvimento da aplicação em si.

Nestas condições, a geração de código e modelos é uma tecnologia que está sendo adotada por todas as empresas que desenvolvem software.

A partir de todos os livros e documentos estudados chega-se a conclusão de que nenhuma ferramenta faz tudo que é necessário. À medida que a MDA for amadurecendo, novos padrões e Frameworks irão surgir fazendo com que as ferramentas sejam melhoradas.

\section{REFERÊNCIAS BIBLIOGRÁFICAS}

DEITEL, D. Internet World Wide Web Como Programar. 2. ed. Porto Alegre: Bookman, 2004.

GONÇALVES, E. Desenvolvendo aplicações Web com JSP, SERVLETS, EJB E AJAX. 1. ed. Rio de Janeiro: Ciência Moderna, 2007.

HERMANAS, P.; CRUZ, S. S. Sistema Gerencial de Representação Comercial. 2006. Trabalho de Conclusão de Curso (Graduação em Sistemas de Informação). Faculdades Integradas "Campos Salles". São Paulo, 2006.

LANO, K. Advanced Systems Design with Java and MDA. 1. ed. Burlington Elsevier, 2005.

LARMAN, C. Utilizando UML e Padrões: uma introdução à análise e projeto orientados a objetos. 2. ed. Porto Alegre: Bookman, 2005.

MACHADO, F. N. R. Banco de dados: projeto e implementação. 2. ed. São Paulo: Érica, 2004.

MAGRI, J. A . e RODRIGUES, V. B. Princípios da Arquitetura Dirigida a Modelos (MDA). 2007. Trabalho de Conclusão de Curso (Graduação em Ciência da Computação) Fundação Instituto Tecnológico de Osasco. Osasco, São Paulo, 2007. 
MELLOR, S. J. MDA Destilada: princípios da arquitetura orientada por modelos. 1. ed. Rio de Janeiro: Ciência Moderna, 2005.

MELO, A.C. Desenvolvendo aplicações com UML. 2. ed. Rio de Janeiro: Brasport, 2005.

NIEDERST, J. Aprenda Web Design. 1. ed. Rio de Janeiro: Ciência Moderna, 2004.

PAULA, W. P. Engenharia de software: fundamentos, métodos e padrões. 2. ed. Rio de Janeiro: LTC, 2005.

RAISTRICK, C. Model Driven Architecture with Executable UML. 1. ed. London: Cambridge University Press, 2004.

ROSEN, M. Which MDA Tools are Right for You? Disponível em: < http:/ www.omg.org/news/meeting /Workshops>. Acesso em: 12 fev 2007

SANTOS, R. R. Programando em Java: teoria e aplicações. 2. ed. Rio de Janeiro: Axcel Books, 2006.

SHEPARD, G. ASP.NET 2.0. Porto Alegre: Bookman, 2006.

SIERRA, K. Head First: EJB. 1. ed. Sebastopol, O' Reilly Inc, 2003. 